Urologe 2019·58:1091-1092

https://doi.org/10.1007/s00120-019-1013-3

Online publiziert: 31. Juli 2019

(c) Springer Medizin Verlag GmbH, ein Teil von Springer Nature 2019

\section{Mein Weg vom Sport in die Medizin}

Ich bin seit 2014 Assistenzärztin im Klinikum Leverkusen. Die Studienzeit habe ich in erster Linie mit Leistungssport, nämlich der Leichtathletik verbracht. Die meisten Kommilitonen kannten mich nur als „die Sportlerin“, richtig kennengelernt habe ich sie größtenteils nicht. Ich musste Seminargruppen tauschen, Klausuren verschieben und schließlich, bedingt durch eingeschobene Urlaubssemester, auch meine Semesterkohorte wechseln.

Nach dem Examen stieg ich zunächst als „Hobbyurologin“ - wie ich liebevoll genannt wurde - mit einer $60 \%$-Stelle ein, damit ich in meinem wirklichen Hobby die zweite Olympiateilnahme realisieren konnte. Nach Beendigung dieser ersten Karriere 2016 sollte es nun in der zweiten, urologischen Karriere $100 \%$ ig weitergehen - nicht nur, was die nun aufgestockte Arbeitszeit betraf.

Im Gegensatz zu den beiden ersten Berufsjahren, die geprägt waren durch Freistellungen für Trainingslager, eine geringere Anzahl an Nachtdiensten, damit die Wettkampfvorbereitung nicht gestört wird und flexiblere Urlaubstage, wenn mal ein Wettkampf kurzfristig einzuplanen war, war ich nun eine "ganz normale Assistenzärztin“.

\section{Das Leben eines Assistenzarztes}

Was erwartet man denn als Assistenzarzt/ ärztin von seinem Job? Ist er wirklich so anstrengend und hart, wie alle sagen? Wie lange dauert es denn überhaupt, ein guter Urologe zu werden? Was ist überhaupt

Linda Stahl

Klinikum Leverkusen, Leverkusen, Deutschland

\title{
Ausbildung junger Assistenzärzte - eine wertvolle Investition in die Zukunft der Urologie
}

ein guter Urologe? Für mich ist ein guter Urologe jemand, der neben einem großen Repertoire an urologischen Eingriffen und einem soliden Fachwissen auch eine herzliche, menschliche Komponente mit einem guten Zugang zu den Patienten vorzuweisen hat. Für das urologische Fachwissen ist jeder selbst verantwortlich und die menschliche Komponente ist eine, die man nur zu einem gewissen Teil erlernen kann. Dieses Repertoire an beherrschten urologischen Eingriffen, das kann und muss man sich hart erarbeiten - es gilt immer noch: „learning by doing“.

Als ungeduldige Leistungssportlerin hatte ich mir natürlich in der Klinik alles etwas schneller und einfacher vorgestellt. Im Alltag muss man Stationsarbeit verrichten, dazu gehören Verbandswechsel, liegengebliebene Blutabnahmen, Angehörigengespräche, viele Arztbriefe und die von jedem Assistenten gefürchteten Nachtdienste. Man schläft weniger, als man es sich in jedem Dienst neu erhofft, und das Telefon klingelt immer dann, wenn man gerade schön weggeschlummert ist - womöglich noch, um die 3-Uhr-Zystitis oder die seit 4 Wochen bestehenden Hodenschmerzen zu sehen.

\section{Operieren ist Trumpf!}

Wer sich als Arzt ein chirurgisches Fachgebiet aussucht, der will operieren!! Also los, dachte ich, worauf warten wir? Die unterschiedlichen OP-Säle in unserem und vermutlich auch jedem anderen Hause haben eines gemeinsam: ein sehr eng getaktetes OP-Programm, zu dem sich jeden Tag noch ungeplante Notfälle gesellen. Zunächst stand Assistieren auf dem Programm, gefühlt unzählige Ma- le mussten mit purer Muskelkraft Haken gehalten werden. Es sieht auch immer so schön einfach aus und es geht dabei noch unglaublich schnell, wie der erfahrene Operateur, der einem gegenübersteht, eine Struktur oder ein Organ freipräpariert. Wird man nun irgendwann selbst zum Operateur, stellt man fest: so einfach ist das gar nicht, auch wenn man am Tag vorher noch einmal theoretisch alles durchgegangen ist und sich einbildet, dass schon alles klappen wird - und man braucht eine gefühlte Ewigkeit für Dinge, von denen man beim 30. Zuschauen gedacht hat „lass mich mal machen“! Niemandem ist die Fähigkeit zu operieren in die Wiege gelegt worden, alles ist eine Frage der Übung und aller Anfang ist schwer. Soweit die Phrasen. Sie entsprechen aber der Wirklichkeit. Und es kostet unglaublich viel Zeit, das Operieren zu lernen. Im Idealfall bedarf es auch einen sehr geduldigen Lehrmeister.

\section{So einfach ist es nicht ...}

Nun sind wir bereits beim Kern des Problems angelangt: Wer hat in der Zeit der Ärzteknappheit, mitten im Überlebenskampf der Krankenhäuser, die Kapazität, mit aller Geduld Assistenten auszubilden? Zeit ist Geld, nicht nur in der Medizin - und dort geht es auch noch um Patientenwohl.

Auf dem 69. Urologenkongress in Dresden 2018 war die Topmeldung, dass die Urologie das größte Zukunftsfach der Medizin ist. Es gibt eine prognostizierte Steigerung des urologischen Versorgungsbedarfes um $20 \%$, was in Zusammenschau mit dem Ärztemangel einen deutlichen Versorgungsengpass 
zur Folge haben wird. Zudem werde die Medizin, und damit auch die Urologie, weiblicher. In der Ärztestatistik von 2016 sind 46,6\% der Ärzte weiblich. Die Anzahl der teilzeitbeschäftigten Ärztinnen ist weiterhin hoch, diese Tatsache verschärft den Ärztemangel über alle Fachbereiche hinweg.

Diese Fakten führen $\mathrm{zu}$ noch engeren Besetzungen in den Kliniken und $\mathrm{zu}$ mehr Arbeit, die auf noch weniger Schultern verteilt wird. Da scheint doch das effektivste Konzept zu sein, jemanden operieren zu lassen, der erfahren, sicher und schnell ist, damit der genau getaktete OP-Plan eingehalten werden kann, es eine hohe Qualität und eine geringe Komplikationsrate für die Patienten gibt. Kurzfristig ist das sehr erfolgsversprechend. Das OP-Personal freut sich über zügige und vermutlich seit etlichen Jahren standardisiert ablaufende Operationen, die Anästhesie ist zufrieden, dass es keine Verzögerungen gibt, die Klinik ist zufrieden mit den Fallzahlen und quasi gleichzeitig wurde von den Assistenzärzten noch die Stationsarbeit erledigt. Doch wie sieht dann die Zukunft aus?

\section{Guter Nachwuchs dringend gesucht}

Schon jetzt stehen die durch den Mangel noch wertvoller gewordenen Assistenzärzte in der Warteschlange, sind motiviert, gute Urologen zu werden. Sie müssen in der bestmöglichen Form praktisch ausgebildet werden, damit sie zum Zeitpunkt der Facharztprüfung eine breite Basis an OP-Fertigkeiten vorzuweisen haben. Zudem können sie auch zu einer Entlastung für die Oberärzte werden, die neben der operativen Tätigkeit noch viele andere Aufgaben in der Klinik erfüllen müssen - jedoch nur, wenn sie so gut ausgebildet wurden, dass sie Eingriffe (zumindest einfachen- und mittleren Schwierigkeitsgrades) auch selbstständig durchführen können. Laut Ärztestatistik 2017 sind 18,4\% der Ärzte älter als 59 Jahre alt - dieses geballte praktische Wissen wird in den kommenden 10 Jahren wegfallen, wenn es nicht vorher an die jüngere Generation weitergegeben wird - zudem wird die Kompetenz im klinischen Alltag fehlen. Genau dann muss die
Folgegeneration über die angesprochene solide Basis verfügen, auf der dann in den nächsten Berufsjahren aufgebaut werden kann. Und um diese herzustellen, kostet es nunmal (OP-)Zeit und Geld. Jedoch gibt es keine wichtigere und lohnendere Investition in die Zukunft als diese.

\section{Was muss bis 2025 passieren?}

Damit die Urologie 2025 ihrer Rolle als zukunftsträchtigstes Fach der Medizin gerecht werden kann, muss noch mehr in die Zukunft investiert werden. Und die Zukunft sind die jungen Ärzte, die ihrer Leidenschaft nachgehen und dafür viele Einschränkungen in ihrer Freizeit in Kauf nehmen- und andererseits auch erwarten, dass man sie zu einem guten Urologen ausbildet.

\section{Was tragen wir dazu bei?}

Ich sehe mich selbst in der Rolle der motivierten, ungeduldigen und wissenshungrigen Assistenzärztin, die lieber gestern als morgen alle Voraussetzungen einer guten Urologin mitbringen würde. Damit bin ich nicht allein. Ich habe nicht nur in der eigenen Klinik, sondern auch auf Kongressen oder Fortbildungen eine Menge junger motivierter angehender Urologen kennengelernt, die alle darauf brennen, von den Chef- oder Oberärzten die Tricks vermittelt zu bekommen, die in keinem Buch stehen und nur durch jahrzehntelange Erfahrung gesammelt werden konnten.

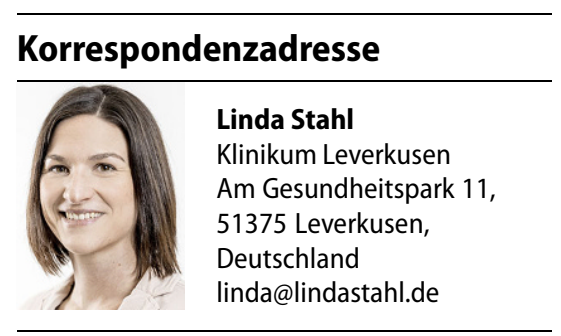

\section{Einhaltung ethischer Richtlinien}

Interessenkonflikt. L. Stahl gibt an, dass kein Interessenkonflikt besteht.

Für diesen Beitrag wurden vom Autor keine Studien an Menschen oder Tieren durchgeführt. Für die aufgeführten Studien gelten die jeweils dort angegebenen ethischen Richtlinien. 